\title{
Same-different discrimination of horizontal and vertical stripe patterns in cats
}

JOHN S. ROBINSON AND DALE R. METZGER, SONOMA STATE HOSPITAL, Eldridge, California, AND THEODORE J. VONEIDA, WESTERN RESERVE UNIVERSITY, Cleveland, Ohio

Cats learned a discrimination task requiring comparison of the orientation of stripe patterns presented in same and different pairs. Horizontal and vertical patterns were back-projected on choice doors, and $S$ received a food reward for selecting the alternative with differently oriented stripes. Controls ruled out response on a configurational basis or in terms of absolute characteristics of the individual patterns.

Over (1967) discusses the inappropriateness of using a recognition method such as a discrimination learning task in the study of a nonverbal S's ability to discriminate stimuli. Such a method involves an associative as well as a discriminative process, since $S$ must learn which stimulus is correct and remember it from trial to trial. If one is interested in S's ability to see the difference or identity of compared stimuli (Robinson \& Higgins, 1967), or in the visual system signals produced by the stimuli (Sutherland, 1963), an oddity, matching or same-different task should be used. Correct response in such tasks requires detection of a stimulus difference (or identity). One of these tasks should also be used when attention is focussed on the comparison process (Robinson \& Voneida, 1955), since consistent correct response guarantees that stimuli are being compared.

Boyd \& Warren (1957) showed that cats can respond to stimulus differences as cues, since they learned a simplified oddity task. Whether or not such a task could be used to study the cat's discrimination of pattern was not demonstrated because the stimuli used also differed in the perceptually more simple brightness and size dimensions.

In this study, cats secured a food reward by responding to cues of sameness and difference in pairs of two-dimensional patterns. They compared the orientation of stripe patterns in choice doors and selected the alternative with the differently oriented patterns (horizontal and vertical) and avoided the door with ientically oriented ones (horizontal-horizontal, vertical-vertical). The task is a true, if limited, discrimination task in that sustained correct performance requires $S$ to compare patterns and to choose (or avoid) a given alternative depending on whether the patterns were seen as different (or the same).

Subjects.

METHOD

The Ss were eight young adult male cats. One $S$ had prior training on a simple horizontal-vertical discrimination problem.

Stimuli

Stripe configurations for the Horizontal-Vertical Same-Different (H-V S-D) task (see Fig. 1) were made into $2 \times 2$ in. slides and back-projected on the milk-glass doors of a two-choice discrimination box. The Ss were trained on four of the eight arrangements possible when the right-left position of the $\mathrm{H}$ and $\mathrm{V}$ components and $\mathrm{S}$ and $\mathrm{D}$ compounds are varied; the other four configurations were used in the control tests described below.

Apparatus and Procedure

Training. Fifteen slides of each of the training configurations were put into the magazine of a Carousel Projector in a 60-trial modified random sequence which was varied from day to day. The $S$ initiated a trial by stepping on a treadle switch in the back of the box; he could then get $.5 \mathrm{cc}$ of strained beef baby food by going to the front and pushing open the correct choice door. A well just outside the door received food through tygon tubing from a $1000 \mathrm{cc}$ reservoir in which it was kept under pressure; opening the correct door resulted in momentary release of a pinch valve (duration of release was controlled by an interval timer), so that $.5 \mathrm{cc}$ of food was foreed into the well. Displacement of the correct door also stepped the projector, but patterns for the next trial were occluded by a shutter. Both doors locked automatically, and the patterns were occluded when an incorrect choice was made; stepping on the rear treadle unlocked the doors and represented the same configuration. This sequence was repeated until $\mathbf{S}$ made a correct choice (only initial choices are reported here).

The Ss were run to a criterion of $90 \%$ correct in one daily 60 -trial session. In the initial stages of training, a brightness difference was added by placing a neutral density filter over the negative stimulus. This difference was gradually reduced as the $S$ showed improvement, and was finally eliminated (two $S$ were given extensive training, short of criterion, on variants of the task before being shifted to the more efficient method described here).

Post criterion tests. Twenty trials with the four configurations not used during training were interspersed with 120 trials with the regular training configurations. Twenty pattern comparison trials were also run (interspersed among 120 standard trials), with right halves or left halves of the choice doors covered. Food was available at either choice door on all test trials.

\section{RESULTS}

Training. Six of the eight Ss reached criterion in 780,876 $1987,2208,8209$ and 9444 trials. (The last two Ss failed to reach criterion on earlier variants of the task, but learned easily when the method described above was used). The remaining two Ss failed to show consistent improvement in 6500 trials. One of them had previously learned a simple horizontal-vertical discrimination with vertical positive. He did very well when the configuration had only one alternative with a vertical component (e.g., HV vs HH), but never exceeded chance on trials when both alternatives had vertical components (e.g., VH vs VV). No such readily identifiable reason could be found for the other S's failure.

Post-criterion tests. Average accuracy on trials with new configurations-Generalization Test trials-was 93\%; on Pattern Comparison trials, with only half of each choice compound visible, accuracy averaged $50 \%$. Average accuracy for concurrently presented standard trials was $92 \%$.

\section{DISCUSSION}

The Ss learned to use the cues of difference or identity of pattern components in a given choice door as a basis for choice. The generalization test trials showed that $S$ had not simply learned specific responses to particular pattern configurations, and the half-door test trials ruled out response on the basis of absolute characteristics of the stimulus components. The H-V S-D task, therefore, qualifies as a true discrimination technique-at least in the context of horizontal and vertical stripes-free of the associative and retention ambiguities of a recognition task. Within the limits of the stimuli used, it is a valid measure of pattern comparison.

Cats learn the H-V S-D task more easily than the oddity problem of Boyd and Warren. A larger proportion of Ss learned the former, and reached a higher terminal performance jevel in fewer trials. This may be because $S$ learns to either approach or avoid a given alternative in H-V S-D, whereas in the oddity problem the alternatives change their value from trial to trial.

The greater ease of learning the S-D task, as compared with the oddity problem, suggests that generalization to new stimuli will occur more readily. The extent of such generalization will determine the usefulness of the task as a method of studying pattern comparison and discrimination in cats or other non-verbal Ss.

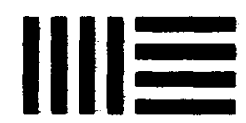
|l||l|||

Fig. 1. Sample H-V S-D configuration. The squure components have seven 3/16-in. stripes. The compound with differently oritnted stripes is positive. 


\section{REFERENCES}

BOYD, B. O., \& WARREN, J. M. Solution of the oddity problem by cats. $J$. comp. physiol Psychol, 1957, 50, 258-260.

OVER, R. Detection and recognition measures of shape discrimination. Nature, 1967, 214, 1272-1273.

ROBINSON, J. S., \& HIGGINS, K. E. The young child's ability to see a difference between mirror-image forms. Percept. mot. Skills, 1967, 25, 893-897.

ROBINSON, J. S., \& VONEIDA, T. J. Central cross-integration of visual inputs presented simultaneously to the separate eyes. $J$. comp. physiol Psychol, 1964, 57, 22-28.

SUTHERLAND, N. S. Shape discrimination and receptive fields. Nature, 1963, 197, 118-122.

\section{NOTE}

1. This work was supported by Grants MH-08832 (JSR) and MH-07051 (TJV), National Institutes of Health, United States Public Health Service, and Grant 67-16-25 (JSR), Department of Mental Hygiene, State of California. 